\title{
Identidad profesional: representaciones sociales de trabajadoras sociales chilenas en tiempos de dictadura
}

\author{
Paula Andrea Opazo-Valenzuela \\ Investigadora autónoma, Chillán, Chile
}

\author{
Carmen Gloria Jarpa-Arriagada \\ Universidad del Bío-Bío, Escuela de Trabajo Social, Chillán, Chile \\ (UBB)
}

\section{Identidad profesional: representaciones sociales de trabajadoras sociales chilenas en tiempos de dictadura}

Resumen: El artículo examina las representaciones sociales de trabajadoras sociales chilenas que se desempeñaron profesionalmente en organismos de defensa de los derechos humanos en el periodo de dictadura cívico-militar chilena. Específicamente, se analizan los componentes centrales en la construcción de su identidad profesional. El estudio es cualitativo y tiene como estrategia de producción de datos la historia de vida temática. Los principales hallazgos indican que los componentes centrales en la construcción de identidad profesional son la formación profesional, la ética y el ejercicio profesional condicionado por el contexto sociohistórico. Se devela una representación social de Trabajo Social comprometido con la defensa de los derechos humanos como un lugar propio, donde el empoderamiento de los sujetos populares fue una característica principal de la cultura de trabajo representada por las trabajadoras sociales entrevistadas.

Palabras clave: Identidad profesional. Trabajo Social. Derechos humanos. Representaciones sociales.

Identidade profissional: representações sociais de assistentes sociais chilenas em tempos de ditadura Resumo: O artigo examina as representações sociais de assistentes sociais chilenas sobre o exercício profissional em organismos de defesa dos direitos humanos no período da ditadura cívico-militar chilena. Especificamente, foram analisados os componentes centrais na construção de sua identidade profissional. O estudo é qualitativo e tem como estratégia de coleta de dados a história de vida temática. Os principais achados indicam que os componentes centrais na construção de identidade profissional são a formação profissional, a ética e o exercício profissional condicionado pelo contexto sócio histórico. O resultado revelou uma representação social do Serviço Social comprometido com a defesa dos direitos humanos como um lugar próprio, onde o empoderamento dos sujeitos populares foi uma característica principal da cultura de trabalho representada pelas assistentes sociais entrevistadas.

Palavras-chave: Identidade profissional. Serviço Social. Direitos humanos. Representações sociais.

Professional Identity: Social representations of Chilean social workers in times of the dictatorship Abstract: This article examines the social representations of Chilean social workers who worked professionally in human rights organizations during Chile's civilian-military dictatorship. It specifically analyzes the central components in the construction of their professional identity. The study is qualitative and its data production strategy involves thematic life histories. The main findings indicate that the central components in the construction of the professional identity are professional education, ethics and professional activity conditioned by the socio-historic context. The article reveals a social representation of social work committed to the defense of human rights as a specific element, in which the empowerment of the popular subjects was a main characteristic of the working culture of the social workers interviewed.

Keywords: Professional identity. Social Work. Human Rights. Social Representations.

Recibido en 20.04.2017. Aprobado en 05.09.2017. Revisado en 04.12.2017.

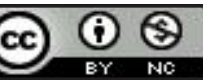

(C) El(Los) Autor(es). 2018 Acceso Abierto Esta obra está licenciada bajo los términos de la Licencia Creative Commons Atribución-NoComercial 4.0 Internacional (https://creativecommons.org/licenses/by-nc/4.0/ deed.es), que permite copiar, distribuir y reproducir en cualquier medio, así como también adaptar, transformar y crear a partir de este material, desde que para fines no comerciales, y que usted fornezca el crédito debido a los autores y a la fuente, insiera un enlace para la Licencia Creative Commons e indique si fueron hechas alteraciones. 


\section{Introducción}

El escenario de violación de derechos humanos y abuso de poder vivenciado en Chile a partir del 11 de septiembre de 1973, alteró rotunda y traumáticamente el rumbo del Trabajo Social. La dictadura chilena "tuvo como práctica habitual y sistemática la violación de los derechos humanos de sus opositores políticos, las que tomaron la forma de desaparición forzada de personas, ejecuciones sumarias, presidio, tortura y exilio" (CÁRDENAS et al., 2013, p. 31). En este contexto, el Trabajo Social como profesión que manifiesta un compromiso ético-político vinculado a la defensa de los derechos humanos (DD.HH), experimentó diversas alteraciones en su quehacer y praxis. Se vio obligada a tomar controversiales decisiones de sobrevivencia tales como retomar viejos saberes ligados al asistencialismo y abandonar por un tiempo indefinido el rol de agente de cambio social que había construido con dedicación y esperanza en la década de los sesenta (CASTAÑEDA; SALAMÉ, 2014). En efecto, el clima socio-político imperante condujo al Trabajo Social chileno a renunciar a la re-construcción de la identidad profesional impulsada en Latinoamérica durante el periodo de reconceptualización. Ciertamente, en la historia del Trabajo Social latinoamericano la reconceptualización constituyó "el primer gran cuestionamiento del Trabajo Social clásico [...] que se inicia a mediados de la década del 60" (AYLWIN, 1999, p. 8) y que, en las circunstancias represivas de los años 1970, ve aplazado su propósito de construir un renovado sentido crítico y reflexivo de la profesión.

\section{[...] respecto a la categoría de} identidad profesional, se evidencia una clara concepción de lo que implica ser trabajador/a social para las entrevistadas, configurada por (1) la formación profesional, (2) las convicciones religiosas $\mathrm{y} / \mathrm{o}$ políticas, (3) el compromiso ético-político para la defensa de los derechos humanos y la transformación de la realidad social.

A pesar de los acontecimientos políticos de la época y la violencia de Estado, algunos trabajadores sociales eligieron dedicar su labor a la defensa de los derechos humanos desafiando al miedo y a la represión ejercida por los organismos de seguridad de la dictadura. Como señalan Castañeda y Salamé (2010), se le concedió énfasis al rol profesional como implementador de políticas sociales, priorizando la atención individual por sobre la atención grupal y comunitaria. No obstante lo anterior, Lira (2010) destaca que tanto la asistencia legal, social, médica como psicológica dirigida hacia las personas perseguidas por la dictadura se organizó muy tempranamente en octubre de 1973, contando con el respaldo de las iglesias y de organismos de solidaridad comprometidos con la defensa de Derechos Humanos. Bajo el mismo propósito, se conforma en el contexto no oficial un colectivo de trabajadores sociales que consolidan sus estrategias de intervención en "el fortalecimiento de las organizaciones, la educación social y cívica, y el apoyo a iniciativas solidarias de las personas y los grupos comunitarios" (CASTAÑEDA; SALAMÉ, 2010, p. 82), recibiendo cooperación internacional a través de organizaciones no gubernamentales y la iglesia católica. Podemos inferir que la fuerte coerción y contracción del campo laboral de Trabajo Social una vez instalada la dictadura no consiguió, en un núcleo importante de profesionales, la adhesión impuesta al régimen de facto, por el contrario, se mantuvo una actitud de rechazo abierto y desafiante al gobierno autoritario.

Por consiguiente, el artículo analiza los componentes centrales de la construcción de identidad profesional en

Trabajadoras Sociales chilenas que se desempeñaron en organismos de defensa de los derechos humanos durante la dictadura cívico-militar. La investigación fue cualitativa ya que el interés fue reconocer las Representaciones Sociales de las profesionales, principalmente, su componente identitario en un contexto de violencia política. La estrategia de recolección de datos fue la Historia de Vida. Varios autores (CASTILLO et al., 2014; CHÁRRIEZ, 2012; FERNÁNDEZ; OCANDO, 2005; VARELA, 2011; VERAS, 2010) coinciden en que las historias de vida son un instrumento potente de conocimiento, que elaboran y transmiten una memoria personal o colectiva de un período histórico concreto, colocando en evidencia el principio sociológico según el cual existe una correspondencia entre lo individual y lo social.

Con el propósito de producir conocimiento situado históricamente, fundado en la experiencia profesional y reflexiva del quehacer profesional, la historia de vida utilizada fue la de tipo temática. De acuerdo a la clasificación de McKerman (2008), esta tipología de historia de vida es aquella que delimita la investigación a 
un tema, asunto o período de vida a través de una exploración a fondo del mismo. Puesto que el interés de la investigación se enfocó en el estudio de las representaciones sociales de trabajadoras sociales sobre su actuar profesional en época de dictadura militar chilena, el método utilizado fue el Análisis Estructural del Discurso.

Martinic (2006) afirma que este tipo de análisis se ha aplicado particularmente en el estudio de representaciones sociales, puesto que propone reglas y procedimientos para definir los principios que las organizan y dan sentido al discurso que los sujetos expresan. Siguiendo a Martinic (1992, 2006), las representaciones sociales son sistemas de referencia de los sujetos, que ordenan las explicaciones sobre hechos y relaciones existentes en el mundo y otorgan lógica y coherencia a la visión que éstos tienen de él. De esta manera, el análisis estructural del discurso permite identificar unidades básicas de significado en la narración de las y los trabajadores sociales, las cuales son denominadas códigos, y las respectivas relaciones existentes entre éstos, que facilitan la comprensión del sentido de sus acciones en el contexto de defensa de los derechos humanos durante la dictadura militar.

El análisis se llevó a cabo a partir del corpus textual de las historias de vida de las trabajadoras sociales participantes de la investigación, obtenido a través de la transcripción de las entrevistas. Para dar cuenta de las unidades de sentido presentes en el texto y de las relaciones existentes entre ellas, primero se establecieron los códigos que orientarían el análisis del discurso enunciado con base en las categorías y subcategorías definidas a priori, procurando mantener cierto rango de flexibilidad para reconocer categorías emergentes.

Se entrevistó un total de siete trabajadoras sociales chilenas, que ejercieron en defensa de los derechos humanos durante la dictadura militar. Las entrevistas se llevaron a cabo en la ciudad de Chillán, Concepción y Santiago de Chile desde el mes de agosto hasta el mes de noviembre de 2016. La producción de datos consideró la ejecución de entrevistas, la transcripción, la construcción de la historia de vida temática y el análisis estructural del discurso.

El presente artículo está estructurado en un desarrollo, dónde nos aproximamos a la contextualización del Trabajo Social en época de dictadura, para luego analizar la Intervención en contexto de violencia política para, finalmente, adentrarnos en los resultados de la investigación. Estos resultados abordan la categoría Identidad Profesional desde dos ámbitos: (1) rol, formación, ética y entorno profesional y (2) ética, formación profesional y roles. Finalizamos con algunas consideraciones finales sobre una temática que nos interpela a revisar nuestro proyecto ético-político y resignificar nuestra identidad profesional en un contexto de gran licuefacción.

\section{Trabajo social chileno y dictadura militar}

La dictadura militar chilena marcó un hito doloroso y significativo en la historia del trabajo social chileno. El gobierno autoritario obstruyó abruptamente los ideales del movimiento de reconceptualización, que inicia a mediados de los años sesenta convocando a la totalidad de escuelas de trabajo social de Latinoamérica, como respuesta a la búsqueda de un modelo de acción que facilitara una real respuesta a los problemas de la región y al clima de reformas sociales y políticas de la época (CASTAÑEDA; SALAMÉ, 2014, 2015; RAMÍREZ, 2004). Como señala Netto (2004), este movimiento fundamentó sus principios y acciones en los aportes de la teoría de la dependencia, del marxismo, de la teología de la liberación y de las propuestas de concientización del pedagogo brasileño Paulo Freire. Así entonces, otorgó una mirada de carácter científico al trabajo social, en tanto acentuó el análisis crítico y situado de su quehacer hasta entonces fuertemente asistencialista, y de los fenómenos sociales a los que se enfrentaba en aquella época.

En primer lugar, el cambio opresivo se reflejó en la formación profesional. Afirman Castañeda y Salamé $(2010,2015)$ que, instalada la dictadura, las mallas curriculares transitaron desde asignaturas vinculadas a técnicas de trabajo con grupos, técnicas de comunicación social, cooperativismo, educación popular y trabajo social comunitario, hacia el rediseño de un plan de estudios reformulado que promovía una formación desideologizada y tecnológica aspirante a la neutralidad en la intervención profesional. Castañeda y Salamé (2010), añaden que los procesos de reforma universitaria, desarrollados a partir de 1967, fueron acallados junto a muchos de sus protagonistas. De esta manera, las demandas del estamento estudiantil por mayor participación en el gobierno universitario y por mayor compromiso institucional con los problemas nacionales fueron abiertamente sofocadas. De las doce escuelas de trabajo social que existían en Chile hasta 1973, pocas sobrevivieron:

En los años sucesivos, las escuelas que permanecieron abiertas suspendieron los ingresos de primer año o restringieron el número de sus vacantes. Se sucedieron expulsiones por sumarios a estudiantes y a equipos académicos y también se produjo el abandono de la universidad por la clandestinidad y el exilio. Quienes permanecieron en la academia debieron comprometerse explícitamente a no involucrase en acciones políticas. (CASTAÑEDA; SALAMÉ, 2014, p. 19). 
Además, aquellos estudiantes que vieron suspendida su formación al inicio de la dictadura militar, tuvieron que cursar nuevamente la carrera con planes especiales de regularización reformulados desde los enfoques clásicos de la profesión. En definitiva, la enseñanza en pos del fortalecimiento de la cohesión social, fue reprimida y desarticulada bajo un limitado y autócrata imperativo de orden social.

Dentro de este marco, el campo laboral de la profesión fue firmemente coaccionado. Al respecto, Castañeda y Salamé (2015) indican, por ejemplo, que en el período pre dictatorial el desempeño del trabajo social se destacaba en el agro chileno, donde la intervención social se basaba en el principio de la educación popular, orientada a la alfabetización, formación y fortalecimiento de la organización, sindicalización y participación campesina. Así, las y los trabajadores sociales acompañaron la toma de decisiones de los grupos y organizaciones en el ámbito productivo, organizacional y comunitario, hasta que, en palabras de Ramírez (2004), fueron relegados nuevamente a la dimensión asistencial de la atención social, enfatizando su rol de implementador de políticas sociales y de administrador de recursos para socorrer a los sectores vulnerables o de extrema pobreza.

En lo que respecta al empleo, Castañeda y Salamé $(2010,2014,2015)$ agregan que además de la censura en la academia y el cuestionamiento en su desempeño, las plazas profesionales para trabajo social fueron contraídas y/o suprimidas, en razón de la declaración de reorganización total de los servicios de administración pública dependientes del Estado. Esta reforma trajo consigo despidos masivos y una importante pérdida de cargos en el histórico y más relevante empleador del trabajo social: el Estado.

\section{Intervención en contextos de violencia política}

La intervención social en contextos de violencia política tiene componentes que la distinguen. En primer lugar, el manejo de la emocionalidad involucrada en el proceso desde el primer contacto con el sujeto de intervención. De acuerdo a Bello (2005), las pérdidas que son consecuencia de la represión política generan una crisis de sentido, puesto que se derrumban bases afectivas y culturales de la existencia individual y colectiva. Varios autores (BELLO, 2005; CORNEJO; ROJAS; BUZZONI, 2007; GUZMÁN, 2001; LIRA, 2010) afirman que en escenarios de violencia, se genera un clima de miedo, de intimidación y de terror que afecta a los sujetos a nivel biológico, psicológico y social, interfiriendo en su libertad de expresión, en sus relaciones interpersonales e incluso en el desarrollo de su ciclo vital. Por lo tanto, en aquel contexto, quienes necesitan o solicitan apoyo profesional son personas, familias y/o comunidades que se encuentran en un estado emocional completamente inestable, con necesidad de apoyo y acompañamiento profesional, en donde la inseguridad y la desconfianza se manifiestan como barreras autodefensivas difíciles de atravesar.

El segundo componente característico de las intervenciones en contexto de violencia política es el carácter de urgencia. Entendiendo que la estabilidad emocional facilita la disposición hacia el cambio, Lira (2013) señala que el actuar profesional en escenarios como el de la dictadura militar chilena, en un inicio se enfoca en aliviar la angustia de los sujetos, a través de la orientación y el apoyo, como una respuesta inmediata ante la emergencia. Al respecto, algunas autoras (BELLO, 2005; LIRA, 2013) plantean la dificultad que presenta el responder de forma eficaz a las necesidades o demandas de las víctimas de violencia, y/o de garantizar la reparación del daño, cuando el contexto que originó la vulneración de derechos se perpetúa. Es decir, si el medio social en el que se desenvuelven las personas violentadas continúa proyectando un alto riesgo de transgresión a su bienestar, la posibilidad de mantener una intervención con objetivos a largo plazo resulta prácticamente inviable.

En esta tarea, se identifican dos nuevos componentes: la multidisciplinariedad y el vínculo profesionalsujeto, que en dictadura militar se denominó vínculo comprometido (GUZMÁN, 2001; LIRA, 2010). A causa de la contingencia, Guzmán (2001) afirma que trabajo social reivindica el método de atención de caso otorgándole una dimensión terapéutica, psicoeducativa y asistencial. La necesidad de direccionar la atención bajo una perspectiva integral del ser humano, impulsa la conformación de equipos de profesionales de diferentes disciplinas, entendiendo que las violaciones de los derechos humanos perturban por completo el bienestar biológico y/o corporal, psicológico y social de las personas.

El quinto y último elemento es la necesidad de autocuidado profesional. Cada integrante de los equipos de trabajo terapéutico, como señala Lira (2010), se ve enfrentado al desgaste emocional debido al impacto de las historias de violencia relatadas por las personas a las que atiende, a la conmoción transferencial y contratransferencial de las experiencias de agresión que los sujetos vivieron, o en ocasiones, a un estado constante de amenaza contra su propia seguridad. Pese a ello, su motivación para involucrarse en este ámbito de intervención, fundada en valores y compromisos históricos, atizaba en ellos una "actitud de omnipotencia frente a este trabajo, como un factor cualitativamente relevante, que facilitaba negar la angustia inherente a la vida propia y al trabajo cotidiano." (LIRA, 2013, p. 22). 


\section{Identidad profesional: rol, formación profesional, ética y entorno profesional}

La identidad es una construcción social que se moldea y transforma dependiendo de los espacios en que los sujetos se desenvuelven. Al respecto, diversos autores (ANDRADE, 2014; AQUÍN, 2003; BOLÍVAR; FERNÁNDEZ-CRUZ; MOLINA, 2005) afirman que la identidad es fruto de procesos de socialización e interacción en contextos sociales y situaciones cambiantes que, a través de identificaciones y atribuciones, configuran en las personas la imagen de sí mismas en función del reconocimiento de los otros. Siguiendo a Hirsch (2013), hablar de identidad profesional es referirse a aquel lugar común compartido entre el individuo, su entorno profesional y social, y la institución en la cual desempeña su labor profesional. Por consiguiente, la identidad profesional es entendida a partir de cuatro elementos particulares: roles, formación, ética y entorno profesional.

De acuerdo a la teoría de identidad social de Scheibe (1998), las subcategorías de estatus, involvement - en adelante implicación - y valoración permiten explorar la categoría de rol. Según el autor, el estatus refiere a las expectativas que son puestas por los miembros de una sociedad sobre determinado rol. Como tal, se evidencia en el reconocimiento por parte de las personas con las que el sujeto se relaciona y la localización social que este posee. Así, se distingue el estatus heredado del estatus adquirido o ganado; el primero corresponde a aquel determinado por las expectativas incluidas en el rol, y el segundo, al generado por la forma en que la persona ha representado su rol. Por otro lado, la implicación alude al grado de vinculación con la actividad que se realiza, patentizada en la inversión de tiempo, esfuerzos y recursos del sujeto en su actuar profesional, o en palabras de Scheibe (1998, p. 95) "la intensidad con que el rol es ejecutado." Finalmente, la subcategoría de valoración da cuenta de los atributos favorables o desfavorables, positivos o negativos que las y los sujetos asignan a su rol. En conclusión, un alto estatus, una alta implicación y una positiva valoración del rol, conllevaría según Scheibe (1998) a una estrecha relación entre rol e identidad. Dicho de otra forma, estas tres dimensiones tienen una relevante y contribuyente función en la representación del ser de los sujetos.

La identidad profesional también tiene una dimensión ética. Durante el proceso de formación profesional suele inculcarse en los estudiantes las distinciones e implicancias de un determinado marco ético de acción y de una serie de principios y normas morales atribuidas a su quehacer profesional. Sin intención de profundizar en la discusión acerca de ética y moral, se entiende su influencia en la construcción identitaria de los trabajadores sociales desde la definición de ética de Ricoeur (2011), quien se refiere a ella como una orientación y respuesta para alcanzar una vida buena con y para otros, mediada por instituciones justas. Duarte (2014) infiere que esta orientación supone reconocer la propia voluntad hacia la vida buena, pero también la de los otros, como portadores de esa misma vocación ética, lo que nos lleva a la reciprocidad, es decir, considerar al otro desde una posición de igualdad. Por lo tanto, la ética sería una orientación global para el bienestar común, y la moral estipularía las disposiciones para lograrlo, dependiendo de cada contexto y sus particularidades.

El entorno profesional está fuertemente vinculado al tipo de trabajo que se realiza, o en el lenguaje de trabajo social, al nivel y área de intervención en que se desempeñan los sujetos. Se ha mencionado previamente, que el elemento entorno contribuye a la construcción de la identidad profesional debido a que esta, como señala Andrade (2014), se concibe como la relación que las personas tienen con su rol ocupacional, y por tanto, con el contexto en el cual se insertan. Estos procesos, de acuerdo a GarcíaLópez; Jover-Olmeda; Escámez-Sánchez (2010), ocurren en las comunidades definidoras, en contextos determinados que dan lugar a distintas experiencias. Por lo tanto, esta concepción de entorno profesional va más allá del espacio físico en el que los profesionales ejecutan sus labores. Alude a la construcción de relaciones desde el discurso, el cual está permeado por cada uno de los componentes culturales, sociales e históricos de la vida de los sujetos.

\section{Identidad profesional en trabajadoras sociales que actuaron en defensa de los DD.HH: ética, formación profesional, roles ejecutados}

La identidad profesional se entiende como una construcción social que se solidifica y moldea con base en las relaciones sociales y las interacciones de los sujetos en distintos contextos y situaciones. Para efectos de este artículo, se analiza la formación profesional desde los valores, la formación y los roles ejecutados como elementos constituyentes de la identidad profesional de las trabajadoras sociales que ejercieron en defensa de los derechos humanos durante la dictadura militar chilena. 
Figura 1: Estructura cruzada 1 - Valores y creencias personales / Conciencia de contexto de riesgo

\begin{tabular}{|c|c|c|c|}
\hline & Opcic & ligiosa & \\
\hline & $\mathbf{A}(++)$ & B (+-) & \\
\hline $\mathbf{A 2}(+)$ & $\begin{array}{c}\text { Trabajadora Social cristiana y } \\
\text { comprometida }\end{array}$ & $\begin{array}{c}\text { Trabajadora Social cristiana y } \\
\text { descomprometida }\end{array}$ & B2 $(-)$ \\
\hline $\begin{array}{c}\text { Actuación profesional } \\
\text { comprometida con DD.HH }\end{array}$ & $\mathrm{C}(+-)$ & D (--) & $\begin{array}{c}\text { Actuación profesional } \\
\text { descomprometida con } \\
\text { DD.HH }\end{array}$ \\
\hline & $\begin{array}{c}\text { Trabajadora Social política y } \\
\text { comprometida }\end{array}$ & $\begin{array}{c}\text { Trabajadora Social, apolítica y } \\
\text { descomprometida }\end{array}$ & \\
\hline & Opci & olítica & \\
\hline
\end{tabular}

Código 1: A1 Opción religiosa / B1 Opción política = T1 Valores y creencias personales.

Código 2: A2 Actuación profesional comprometida con DD.HH / B2 Actuación profesional descomprometida con DD.HH = T2 Conciencia de contexto de riesgo.

Fuente: Figura elaborada por las autoras.

Como se observa en la Figura 1, dentro de cada cuadrante se ha definido un concepto que nace de la vinculación de las categorías de análisis que conforman las respectivas totalidades o códigos. Cada una de estas categorías representan el signo $(+)$ o (-) dependiendo de su posición en la construcción de estructuras paralelas elaboradas a partir del relato de las participantes, extraído del corpus de las entrevistas. Cabe resaltar que las valoraciones de los códigos $(+)$ y (-) dan cuenta de la distinción entre ellos, como inversos y/o opuestos. Así, de las categorías resultantes del cruce entre Valores y creencias personales y Conciencia de contexto de riesgo, hay una Trabajadora Social en el cuadrante A (++) Trabajadora social cristiana y comprometi$d a$; tres pertenecerían al cuadrante C (+-) Trabajadora social política y comprometida; y dos se ubicarían entre ambos cuadrantes A (++) Trabajadora social cristiana y comprometida y C (+-) Trabajadora social política y comprometida, ya que manifiestan una postura política clara (mas no militancia) y al mismo tiempo reconocen influencia religiosa en su decisión de actuar en defensa de los derechos humanos durante el contexto histórico estudiado.

Desde el punto de vista del Construccionismo Social de Gergen (2006), la identificación de las participantes con una creencia religiosa o una postura política, dotaría de sentido su actuar profesional desinteresado y comprometido. Existen valores socialmente construidos y atribuidos a cada opción religiosa o política que, según la mayoría de las entrevistadas, son coherentes con los principios y valores del Trabajo Social, como el respeto por la dignidad humana, el actuar ante la injusticia o el resguardo de la democracia. Sin duda, la construcción colectiva influiría en la convicción individual de las entrevistadas de ejercer en defensa de los derechos humanos de las víctimas de violencia política, asumiendo todo el riesgo del contexto en el cual estaban insertas.

En la Figura 2 se presenta la estructura cruzada conformada por las totalidades Formación profesional y Convicción ética. En el cuadrante C (+-) Trabajadora social crítica con convicción ética se identifica a cinco trabajadoras sociales. Si bien se formaron en distintas escuelas, en general su formación estuvo influida por el movimiento de reconceptualización del trabajo social y las propuestas ideológicas de la Educación Popular de Paulo Freire. Por otro lado, una pertenecería al cuadrante A (++) Trabajadora social tradicional con convicción ética ya que su periodo de formación fue en los inicios de los años 1950, donde el trabajo social tenía una fuerte concepción aséptica-tecnocrática. Sin embargo, sus convicciones valóricas y compromiso ético-político evidenciado en su desempeño profesional, dan muestra de su convicción ética. Finalmente, una profesional se ubicaría entre el cuadrante A (++) Trabajadora social tradicional con convicción ética y C (+-) Trabajadora social crítica con convicción ética, puesto que si bien su formación en México estuvo enfocada a la solidaridad y no tuvo un componente netamente político, sí estuvo orientada a la defensa de los derechos humanos en el contexto de las dictaduras militares que se impusieron en Latinoamérica durante aquel período histórico. 
Figura 2: Estructura cruzada 2 - Formación profesional / Convicción ética

\begin{tabular}{|c|c|c|c|}
\hline & Formación prof & $\begin{array}{l}+\infty \\
\text { ional tradicional }\end{array}$ & \\
\hline & $\mathbf{A}(++)$ & B (+-) & \\
\hline $\mathbf{A 2}(+)$ & $\begin{array}{c}\text { Trabajadora social tradicional con } \\
\text { convicción ética }\end{array}$ & $\begin{array}{c}\text { Trabajadora social tradicional sin } \\
\text { convicción ética }\end{array}$ & B2 (-) \\
\hline $\begin{array}{l}\text { Presencia de } \\
\text { convicción } \\
\text { Ética }\end{array}$ & $C(+-)$ & D (--) & $\begin{array}{l}\text { Ausencia de } \\
\text { convicción } \\
\text { Ética }\end{array}$ \\
\hline & $\begin{array}{l}\text { Trabajadora social crítica con } \\
\text { convicción ética }\end{array}$ & $\begin{array}{l}\text { Trabajadora social crítica sin } \\
\text { convicción ética }\end{array}$ & \\
\hline & $\begin{array}{r}\text { B } \\
\text { Formación pr }\end{array}$ & $\begin{array}{l}(-) \\
\text { esional crítica }\end{array}$ & \\
\hline
\end{tabular}

Código 1: A1 Formación profesional tradicional/B1 Formación profesional crítica=T1 Formación profesional.

Código 2: A2 Presencia de convicción ética/B2 Ausencia de convicción ética=T2 Convicción ética.

Fuente: Figura elaborada por las autoras.

Por lo tanto, la formación profesional se consolida como un componente fundamental orientador del actuar profesional de las trabajadoras sociales que ejercieron en defensa de los derechos humanos, sin embargo, otros componentes como los valores y principios religiosos, y la postura ético-política personal y/o profesional configurados por el contexto familiar, social e histórico, juegan también un papel relevante en su configuración identitaria.

Los roles profesionales profundizan el estudio de la identidad profesional, puesto que como patrones normativos culturales atribuidos a una posición social, configuran expectativas sobre el actuar profesional que se cumplen o no según la identificación de los profesionales con los mismos. En esta investigación, estos se entienden en función de tres dimensiones: (1) estatus, heredado o adquirido, dado por las expectativas puestas sobre el desempeño del rol, (2) implicación en el desarrollo del rol profesional y (3) valoración del rol profesional ejecutado en la defensa de los derechos humanos.

Figura 3: Estructura cruzada 3 - Asistencialismo en intervención en DD.HH / Rol trabajo social en defensa de DD.HH

\begin{tabular}{|c|c|c|c|}
\hline & Asistencialismo como & io para potenciar lazos & \\
\hline & $\mathbf{A}(++)$ & B (+-) & \\
\hline A2 (+) & Trabajo Social emancipador & Trabajo Social conservador & B2 (-) \\
\hline Rol crítico-orientador & $C(+-)$ & D (- -) & autoritario \\
\hline & Trabajo Social funcional & Trabajo Social dominador & \\
\hline & & & \\
\hline
\end{tabular}

Asistencialismo como fin en sí mismo

Código 1: A1 Asistencialismo como un medio para potenciar lazos / B1 Asistencialismo como fin en sí mismo=T1 Concepción de asistencialismo en intervención en DD.HH.

Código 2: A2 Rol crítico-orientador/B2 Rol indiferente-autoritario= T2 Rol del trabajo social en defensa de los DD.HH.

Fuente: Figura elaborada por las autoras. 
En este caso, la totalidad de las trabajadoras sociales entrevistadas se ubica en el cuadrante A $(++)$ Trabajo social emancipador. Todas se caracterizan por mantener un rol activo y orientador, de acompañamiento, y altamente confiable para los sujetos de intervención. Se presume, que la utilización del asistencialismo como estrategia para la formación de grupos tuvo directa influencia de paradigmas y/o corrientes emancipadoras que resonaron con fuerza durante el movimiento de reconceptualización del Trabajo Social en Latinoamérica, periodo en el que estudiaron y/o ejercieron las siete trabajadoras sociales.

En sus discursos, ellas hacen una alta valoración al trabajo comunitario como la única herramienta capaz de generar cambios, potenciar lazos sociales y enfrentar las problemáticas sociales. Asimismo, en la conformación de grupos de apoyo y de trabajo en tiempos de dictadura, consideran que los y las trabajadoras sociales desempeñaban principalmente un rol de acompañamiento, pues reconocían a las personas como protagonistas en la transformación social. Por último, desde su perspectiva, los sujetos y los otros profesionales reconocían que el Trabajo Social tenía un rol muy importante al interior de las instituciones de defensa de los derechos humanos, por generar un espacio para la contención emocional, el apoyo y la orientación para hacer frente a cada problemática con sus respectivas particularidades.

Figura 4: Estructura cruzada 4 - Implicación en el rol de defensa de DD.HH / Valoración del rol de defensa de DD.HH

\begin{tabular}{|c|c|c|c|}
\hline \multirow{6}{*}{$\begin{array}{c}\mathrm{A2}(+) \\
\text { Experiencia Positiva }\end{array}$} & \multicolumn{2}{|c|}{$\mathrm{A} 1(+)$} & \multirow{5}{*}{$\begin{array}{c}\text { B2 (-) } \\
\text { Experiencia Negativa }\end{array}$} \\
\hline & $\mathbf{A}(++)$ & B (+-) & \\
\hline & $\begin{array}{l}\text { Vinculación rol-identidad } \\
\text { comprometida satisfactoria }\end{array}$ & $\begin{array}{l}\text { Vinculación rol-identidad } \\
\text { comprometida insatisfactoria }\end{array}$ & \\
\hline & $\mathrm{C}(+-)$ & D (- -) & \\
\hline & $\begin{array}{l}\text { Vinculación rol-identidad } \\
\text { indiferente satisfactoria }\end{array}$ & $\begin{array}{l}\text { Vinculación rol-identidad } \\
\text { indiferente insatisfactoria }\end{array}$ & \\
\hline & 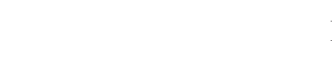 & $-\Rightarrow$ & \\
\hline
\end{tabular}

Código 1: A1 Alta implicación en el rol/B1 Baja implicación en el rol=T1 Implicación en el rol de defensa de DD.HH. Código 2: A2 Experiencia positiva/B2 Experiencianegativa = T2 Valoración del rol de defensa de DD.HH.

Fuente: Figura elaborada por las autoras.

En la Figura 4 se cruzan las totalidades Implicación en el rol de defensa de DD.HH y Valoración del rol de defensa de DD.HH. Entendiendo que según Scheibe (1998) tanto el estatus, como la implicación y la valoración del quehacer profesional son dimensiones del rol, el dar cuenta de un alto estatus como el que manifiestan las entrevistadas, una alta implicación y una positiva valoración del rol, desencadenaría una estrecha relación entre rol e identidad. En este sentido, seis trabajadoras sociales se encontrarían en el cuadrante $\mathrm{A}(++)$ Vinculación rol-identidad comprometida satisfactoria puesto que, pese a manifestar presencia de dificultades en su quehacer profesional, ven su experiencia como altamente positiva. Por otro lado, una profesional estaría ubicada entre el cuadrante A (++) Vinculación rol-identidad comprometida satisfactoria y B (+-) Vinculación rol-identidad comprometida insatisfactoria. En efecto, pese a considerar su experiencia en defensa de los derechos humanos altamente satisfactoria y positiva, manifiesta haber sufrido frustración profesional, aislamiento profesional y un importante daño laboral durante un tiempo prolongado.

En suma, las trabajadoras sociales entrevistadas se identifican fuertemente con el rol que desempeñaron en la defensa de los derechos humanos durante la dictadura militar chilena, destacándose el compromiso éticopolítico, independiente de las condiciones de riesgo personal-social a las cuales se enfrentaban. Rescatan la experiencia como un importante y enriquecedor aprendizaje, un privilegio que les brindó la posibilidad de salvar vidas y ser parte activa de aquel período histórico de Chile. 


\section{Consideraciones finales}

Desde una perspectiva global, se pueden apreciar varios puntos en común en las historias de vida temática y en el análisis estructural del discurso de las trabajadoras sociales. Estos dan cuenta de las representaciones sociales que han configurado aspectos relevantes de la identidad profesional de quienes ejercieron en defensa de los derechos humanos durante la dictadura militar chilena.

En primer lugar, respecto a la categoría de identidad profesional, se evidencia una clara concepción de lo que implica ser trabajador/a social para las entrevistadas, configurada por (1) la formación profesional, (2) las convicciones religiosas y/o políticas, (3) el compromiso ético-político para la defensa de los derechos humanos y la transformación de la realidad social. En suma, la razón de ser y propósito de nuestra profesión, independiente del contexto histórico o social en el que los/as profesionales estén insertos, se ancla en territorios de densidad profunda como parte del estatus adquirido durante la trayectoria formativa y profesional de los trabajadores sociales. Impera una consistente representación social de un trabajo social fuertemente comprometido con el cuestionamiento de las problemáticas sociales en pos del bienestar común por sobre el individual. Al mismo tiempo, frente a contextos de violencia política, se considera que los/as trabajadores/as sociales han de asumir y enfrentar las condiciones de riesgo a las que pueden estar expuestos/as.

La relevancia del estatus y la alta implicación y valoración que las trabajadoras sociales demuestran en su ejercicio profesional durante el período de dictadura militar traduce una fuerte identificación con el rol desarrollado durante aquella época. De la misma forma, esta concepción del trabajo social implica, en referencia a la categoría de roles, una delimitación que configura una distinción con otras profesiones, con las cuales se desarrolló un importante trabajo interdisciplinario durante la dictadura: la relación cercana y comprometida con las personas y familias, la potenciación de lazos sociales a través de la organización grupal-comunitaria, la visión de los sujetos de intervención como actores sociales protagonistas con capacidades y habilidades, la utilización de estrategias para una intervención social en función de construir una sociedad democrática y justa, son características del quehacer propio de las trabajadoras sociales participantes en esta investigación.

\section{Referencias}

ANDRADE, V. Identidad profesional y el mundo del trabajo contemporáneo. Reflexiones desde un resumen de caso. Athenea digital: Revista de pensamiento e investigación social, Bellaterra (España), v. 14, n. 2, p. 117-145, 2014.

AQUÍN, N. El trabajo social y la identidad profesional. Prospectiva: Revista de Trabajo Social e Intervención Social, Cali (Colombia), n. 8, p. 99-110, 2003.

AYLWIN, N. Identidad e historia profesional. Revista Colombiana de Trabajo Social, Cali (Colombia), n. 13, p. 7-23, 1999.

BELLO, M. N. Trabajo Social en contextos de violencia política. Trabajo Social, Bogotá, n. 7, p. 9-20, 2005.

BOLIVAR, A.; FERNÁNDEZ-CRUZ, M.; MOLINA, E. Investigar la identidad profesional del profesorado: una triangulación secuencial. Forum: Qualitative Social Research, v. 6, n. 1, p. 1-26, 2005.

CÁRDENAS, M. et al. Emociones como predictores del perdón en el contexto de la violación a los derechos humanos en Chile. Psicoperspectivas: Individuo y Sociedad, Viña del Mar (Chile), v. 12, n. 1, p. 30-49, 2013.

CASTAÑEDA, P.; SALAMÉ, A. M. Memoria profesional y Trabajo Social chileno. Reforma agraria y dictadura militar. Revista Katálysis, Florianópolis, v. 18, n. 2, p. 258-266, 2015.

(Chile), n. 8, p. 68-92, 2010

Perspectiva histórica de la formación en Trabajo Social en Chile. Revista electrónica de Trabajo Social, Concepción

.; ___ Trabajo Social chileno y dictadura militar. Memoria profesional predictatorial periodo 1960-1973. Agentes de cambio y trauma profesional. Rumbos TS: Un espacio crítico para la reflexión en Ciencias Sociales, Santiago, n. 9, p. 8-25, 2014.

CASTILLO, A. et al. La historia de vida como instrumento de aprendizaje del trabajo social. In: PASTOR SELLER, E. et al. (Coord.). El trabajo social ante el reto de la crisis y la educación superior. Murcia: Universitas, 2014. p. 113-120.

CHÁRRIEZ, M. Historias de Vida: Una metodología de investigación. Revista Griot, San Juan (Puerto Rico), v. 5, n. 1, p. 50-67, 2012. CORNEJO, M.; ROJAS, R.; BUZZONI, M. E. Prisión política y tortura: desde las intervenciones psicosociales a las políticas de reparación. Persona y Sociedad, Santiago, v. 21, n. 1, p. 59-81, 2007.

DUARTE, C. La formación en Derechos Humanos como parte del proyecto ético político del Trabajo Social. Serviço Social \& Sociedade, São Paulo, n. 119, p. 482-507, jul./set. 2014.

FERNÁNDEZ, O.; OCANDO, J. La búsqueda del conocimiento y las historias de vida. Revista Omnia, Maracaibo (Venezuela), v. 11, n. 1, p. 1-14, 2005

GARCÍA-LÓPEZ, R.; JOVER-OLMEDA, G.; ESCÁMEZ-SÁNCHEZ, J. Ética profesional docente. Madrid: Síntesis, 2010.

GERGEN, K. Construir la realidad: el futuro de la psicoterapia. Barcelona: Paidós, 2006. 
GUZMÁN, J. M. Trabajo Social y Derechos Humanos. Reflexión: Revista del Centro de Salud Mental y Derechos Humanos, Santiago, v. 25 , p. $1-4,2001$.

HIRSCH, A. Elementos teóricos y empíricos acerca de la identidad profesional en el ámbito universitario. Perfiles educativos, v. 35, n. 140, p. 63-81, 2013.

LIRA, E. Algunas reflexiones a propósito de los 40 años del golpe militar en Chile y las condiciones de la reconciliación política. Psykhe, Santiago, v. 22, n. 2, p. 5-18, nov. 2013.

. Trauma, duelo, reparación y memoria. Revista de Estudios Sociales, Bogotá, n. 36, p. 14-28, 2010.

MARTINIC, S. Análisis Estructural: presentación de un método para el estudio de lógicas culturales. Santiago: Centro de Investigación y Desarrollo de la Educación, 1992.

El estudio de las representaciones y el Análisis Estructural de Discurso. In: CANALES, M. (Ed.). Metodologías de investigación social: introducción a los oficios. Santiago: Lom, 2006. p. 299-319.

MCKERMAN, J. Investigación-acción y curriculum: métodos y recursos para profesionales reflexivos. Madrid: Morata, 2008.

NETTO, J. P. Trabajo Social de cara a la realidad latinoamericana. Prospectiva: Revista de Trabajo Social e Intervención Social, Cali (Colombia), n. 9, p. 13-19, 2004.

RAMÍREZ, F. Adiós “Señorita Asistente”. Construyendo la historia del Trabajo Social en Chile. Revista de Ciencias Sociales, Tarapacá (Chile), n. 14, p. 129-135, 2004.

RICOEUR, P. Ética y moral. In: GÓMEZ, C. (Ed.). Doce textos fundamentales de la ética del siglo XX. Madrid: Alianza, 2011. p. 241-255. SCHEIBE, K. Self studies: the psychology of self and identity. Westport, CT: Praeger Publishers, 1998.

VARELA, J. Historias de Vida: La crisis del mundo rural. In: GORDO, A. J.; SERRANO, A. (Coord.). Estrategias y prácticas cualitativas de investigación social. México: Pearson Educación, 2011. p. 189-210.

VERAS, E. Historia de Vida: ¿Un método para las ciencias sociales? Cinta de Moebio, Santiago, n. 39, p. 142-152, dic. 2010.

\section{Paula Andrea Opazo-Valenzuela}

pauopazov@gmail.com

Licenciatura en Trabajo Social por la Universidad del Bío-Bío (UBB)

\section{Carmen Gloria Jarpa-Arriagada}

cjarpa@ubiobio.cl

Doctorado en Ciencias de la Educación por la Universidad de La Frontera (UFRO)

Académica de la Escuela de Trabajo Social de la Universidad del Bío-Bío (UBB)

\section{UB B}

Avenida Brasil, 1180

Chillán - Provincia de Nuble - Chile

Código Postal: 3780000 